\title{
The entropy weight fuzzy comprehension evaluation of enterprise knowledge competitiveness
}

\author{
YU Lei, WANG Shu-zhen \\ (Department of Business, Agriculture University of Hebei, Baoding 071001, China)
}

\begin{abstract}
With the constant development of knowledge-based economy, knowledge has become the core competitiveness of enterprise, which plays a vital role in the development of enterprise and market competition. It will be helpful for enterprise managers to improve their knowledge competitiveness, if they can judge the advantage and disadvantage of knowledge competitiveness reasonably. According to the nature and the characteristic of knowledge competitiveness of enterprise, a system of evaluation index has been designed about the knowledge competitiveness of enterprise. Taking the enterprise $A$ as an example, it is appraised by using fuzzy comprehension evaluation based on entropy weight, and has provided a new idea for the knowledge competitiveness of enterprise.
\end{abstract}

Key words: knowledge competitiveness; entropy weight; fuzzy comprehension evaluation

With the constant development of knowledge-based economy, the competition in business had become the competition of knowledge. Reasonable methods and index system which can judge the knowledge competitiveness of enterprise would be helpful for managers to discover the advantages and disadvantages. It is especially important for some Chinese enterprises, as most enterprises were still in the stage of capital-intensive, which needed to improve the knowledge competitiveness greatly.

\section{The evaluation index system about the knowledge competitiveness of enterprise}

\subsection{The design principles of the evaluation index system}

\subsubsection{The scientific principles}

The scientific index system is the basis which may ensure that evaluative results were accurate and reasonable. The elements of knowledge competitiveness and reasonable structure of index system must be taken into consideration, when designing the evaluation index system about knowledge competitiveness of enterprise. The index system ought to reflect the knowledge competitiveness of enterprises at different angles and should be reliable, independent and representative.

\subsubsection{The comprehensive principle}

The knowledge competitiveness of enterprises is a multiple system which composed by many elements, which must learnt from different angles. Therefore, the design of the index system must be done from the overall system, and then select the relevant indicators using the system analysis method in order to reflect the basic

YU Lei, Ph.D. candidate, Department of Business, Agriculture University of Hebei; research fields: management, accounting and appraisal.

WANG Shu-zhen, professor, Department of Business, Agriculture University of Hebei; research fields: management, accounting and appraisal. 
features of knowledge competitiveness comprehensively and scientifically.

\subsubsection{The relative independence principle}

The indicators must maintain the relative independence under the premise of the comprehensive principle, and should avoid the phenomenon of inclusion, cross and kindred among them. Thus, they can reflect knowledge competitiveness of enterprises objectively.

\subsubsection{The operational principle}

In the process of selecting indicators, we must ensure the feasibility for the selected indicators and should do our best to choose the quantitative indicators and avoid the qualitative indicators which can't be identified easily.

\subsubsection{The continuity principle}

In order to avoid some of the abnormal findings, we should select the continuity and stability indicators in accordance with the indicators' historical trend which can keep the indicators scientifically.

\subsection{Establish the evaluation index set}

The evaluation factors set $(U)$ is the set of the comprehensive evaluation indicators, it is layered, namely, the first level indicators $U=\left\{U_{1}, U_{2}, U_{3}, U_{4}, U_{5}\right\}$, the second level indicators is $U_{i}=\left\{U_{i 1}, U_{i 2}, \cdots, U_{i j}\right\}$, in the formula, $U_{i j}$ denotes the $j$-th indicator in the $i$-th layer.

\subsection{Establish the evaluation index system}

The evaluation index system of knowledge competitiveness was composed by key factors which decide the knowledge competitive ability. By analyzing the constitute elements and system structure of knowledge competitiveness of enterprises, it can be divided into four branches: technology factor, brand factor, resource factor and organization factor, which composed the first level indicators of the evaluation index system. If fractionizing the first level indicators, we can gain more specific indexes, which are the second level indicators.

\subsubsection{Technology factor}

(1) Research and development (R\&D) expense ratio

$R \& D$ expense ratio reflected the proportion of capital investment in $R \& D$.

(2) Rate of invention put into product

This indicator reflected the ability of enterprise to transform the invention into economic value.

(3) Patent occupancy

Patent occupancy represented capacity of the enterprise for technological innovation.

(4) Technology value-added rates of product

Technology value-added rates of product reflected the contribution of technology to the profit margin.

\subsubsection{Brand factor}

(1) The fame of brand

The fame of brand was market fame of brand and value of brand, which was accumulated in the process of market operation (SU Yong, 2002).

(2) The kinds of trademark

Different kinds of trademark had the different brand values. Generally speaking, trademark can be classified as ordinary trademark, famous trademark, well-known trademark and time-honored trademark.

(3) Market share

Market share of the enterprise could reflect consumers' recognition of the trademark to a certain extent.

1.3.3 Resources factor 
Resources factor contains the following:

(1) Staff quality; (2) Staff loyalty; (3) Managers' intuitivism; (4) Marketing network; (5) Customer resources. 1.3.4 Organization

These factors are:

(1) Enterprise system; (2) Managerial effectiveness; (3) Managers' innovation consciousness; (4) Corporate culture; (5) Information negotiability (SU Yong, 2002; LI Jin, 2005).

\section{The entropy weight fuzzy comprehensive evaluation model}

\subsection{To ascertain the indicators weight}

\subsubsection{Assumptions}

Assumption: There are $m$ score people and $n$ evaluation indexes. $X_{i} k$ denotes the actual value of the score people $(\mathrm{k})$ to the $i$-th indicator in the e-commerce enterprises. $x_{i}{ }^{*}$ is the ideal value (maximum value) of the indicator $i$. If the evaluation data is positive, $x_{i}{ }^{*}$ is the maximum value in this group data. Similarly, if the evaluation data is negative, $x_{i}{ }^{*}$ is the minimum value in this group data. In data processing, the reverse indicator and the moderation indicator will be translated into positive indicator, therefore, the ideal value $\left(x_{i}^{*}\right)$ in the paper is the maximum value.

2.1.2 Define the proximity degree $D_{i} k$, and unitary treatment it to $d_{i} k$

$D_{i} k$ is the proximity degree of $x_{i}^{*}$ to $x_{i} k$. When $x_{i}^{*}$ is a positive indicator, $D_{i} k=\frac{x_{i} k}{x_{i}^{*}}, x_{i}^{*}=\max \left\{\mathrm{x}_{\mathrm{i}} \mathrm{k}\right\}$. Unitary treatment $D_{i} k$, namely, $d_{i} k=\frac{D_{i} k}{\sum_{i=1}^{n} \sum_{k=1}^{n} D_{i} k}$, make $0 \leq d_{i} k \leq 1, \sum_{i=1}^{n} \sum_{k=1}^{n} d_{i} k=1$.

2.1.3 Calculated entropy value e $\left(d_{i}\right)$ :

$$
e\left(d_{i}\right)=-\frac{1}{\ln m} \sum_{k=1}^{m} \frac{d_{i} k}{\sum_{k=1}^{m} d_{i} k} \ln \frac{d_{i} k}{\sum_{k=1}^{m} d_{i} k}, 0 \leq e\left(d_{i}\right) \leq 1 .
$$

2.1.4 Ascertain the weight of the evaluation indicators $\left(\theta_{t}\right)$ based on the entropy value:

$$
\theta_{l}=\frac{1}{n-E_{e}}\left[1-e\left(d_{i}\right)\right], \text { and } 0 \leq \theta_{\iota} \leq 1, \sum_{i=1}^{n} \theta_{l}=1
$$

\subsection{Establish the remark set}

The remark set is the set of the probable results about the various indicators. We can invite the related field experts to assess the outcomes. Considering of the overall competitive ability in e-commerce enterprises, the authors establish the remark set about the evaluation indicators: $V=\left\{V_{1}\right.$ (strong competition), $V_{2}$ (relative strong competition), $V_{3}$ (general competition), $V_{4}$ (weak competition) $\}$.

\subsection{Ascertain the weight set}

The weight set of the first level indicators is $W=\left\{W_{1}, W_{2}, W_{3}, W_{4}, W_{5}\right\}$.

The weight set of the second level indicators is $W_{i}=\left\{W_{i 1}, W_{i 2}, \cdots, W_{i j}\right\}$.

\subsection{Establish the evaluation matrix}




$$
R=\left[\begin{array}{l}
R_{1} \\
R_{2} \\
\vdots \\
R_{m}
\end{array}\right]=\left[\begin{array}{cccc}
r_{11} & r_{12} & \cdots & r_{1 n} \\
r_{21} & r_{22} & \cdots & r_{2 n} \\
\vdots & \vdots & \vdots & \vdots \\
r_{m 1} & r_{m 2} & \cdots & r_{m n}
\end{array}\right]
$$

In the formula, $R i$ denotes the evaluation structure to the factors $i ; r_{i j}$ denotes the subject degree of evaluation factor $i$ to the evaluation degree $j$, and it denotes the fuzzy relation between the evaluation factor and the evaluation degree. $N$ denotes the amount of evaluation degree in remark set, $m$ denotes the amount of the evaluated factors.

\subsection{The multilevel fuzzy comprehensive evaluation}

Firstly, we go along the first level fuzzy comprehensive evaluation, and calculate the indicators weight (W) and the evaluation matrix (R) on the basis of entropy weight evaluation method, then we use the fuzzy algorithm to carry through the integrated operations and unitary treatment, and finally gain the subject vector $\left(\mathrm{S}_{\mathrm{i}}\right)$ of factor $U_{i}$ to the remark set $V$ (ZHOU Jian-guo \& NIU Dong-xiao, 2004).

$$
S_{i}=W_{i} \times R_{i}=\left(W_{i 1}, W_{i 2}, \cdots, W_{i j}\right) \times\left[\begin{array}{cccc}
r_{11} & r_{12} & \cdots & r_{1 n} \\
r_{21} & r_{22} & \cdots & r_{2 n} \\
\vdots & \vdots & \vdots & \vdots \\
r_{m 1} & r_{m 2} & \cdots & r_{m n}
\end{array}\right]
$$

Then, we go along the second level fuzzy comprehensive evaluation, gain the total evaluation vector $A$ and the comprehensive evaluation conclusion.

$$
A=W \times S
$$

\section{Empirical analysis}

PL was a major pharmacy company in northern China. Vitamin and antibiotic were main products of PL. This paper took PL Company as the object of study and wanted to evaluate the knowledge competitiveness of PL Company using the evaluation method of fuzzy comprehension based on entropy weight. According to the indicator system, the authors design the questionnaire for the four degree of all the indicators. The respondents included managers of PL Company, employees of PL Company, customers and government officer of intellectual property, whose ratio was 4:3:3:1. To make a calculation of some relevant indicators, we also made an elaborative analysis of financial statements of PL Company. According to the above data, we could get the important evaluation of evaluation people to the indicators, then we could get the indicator weight by the entropy weight evaluation method and the remark set through the questionnaire.

According to questionnaire and equation (1), we go along with the fuzzy comprehensive evaluation:

$$
S_{1}=W_{1} \times R_{1}=\left[\begin{array}{c}
0.32 \\
0.26 \\
0.28 \\
0.14
\end{array}\right]^{T} \times\left[\begin{array}{cccc}
0.49 & 0.41 & 0.1 & 0 \\
0.35 & 0.30 & 0.20 & 0.15 \\
0.28 & 0.29 & 0.27 & 0.16 \\
0.45 & 0.40 & 0.10 & 0.05
\end{array}\right]=\left[\begin{array}{c}
0.39 \\
0.35 \\
0.17 \\
0.09
\end{array}\right]^{T}
$$

Similarly, $\quad S_{2}=W_{2} \times R_{2}=\left[\begin{array}{llll}0.40 & 0.36 & 0.16 & 0.08\end{array}\right] \quad ; \quad S_{3}=W_{3} \times R_{3}=\left[\begin{array}{llll}0.32 & 0.32 & 0.28 & 0.08\end{array}\right]$; 


$$
S_{4}=W_{4} \times R_{4}=\left[\begin{array}{llll}
0.24 & 0.46 & 0.25 & 0.05
\end{array}\right] \text { are available. }
$$

With the second fuzzy evaluation using the equation (2), the final evaluation results are available:

$$
A=W \times S=W \times\left[\begin{array}{l}
S_{1} \\
S_{2} \\
S_{3} \\
S_{4}
\end{array}\right]=\left[\begin{array}{l}
0.32 \\
0.16 \\
0.29 \\
0.23
\end{array}\right] \times\left[\begin{array}{llll}
0.39 & 0.35 & 0.17 & 0.09 \\
0.40 & 0.36 & 0.16 & 0.08 \\
0.32 & 0.32 & 0.28 & 0.08 \\
0.24 & 0.46 & 0.25 & 0.05
\end{array}\right]=\left[\begin{array}{c}
0.37 \\
0.33 \\
0.22 \\
0.08
\end{array}\right]
$$

In order to compare them conveniently, the authors translated the comprehensive evaluation results into the score and adopt the evaluation degree $V=\{$ strong, relative strong, general, weak $\}=\{90,80,70,60\}$. In the evaluation of knowledge competitiveness of PL company, the score of the second level evaluation findings is:

$$
U_{1}=S_{1} \times V=0.39 \times 90+0.35 \times 80+0.17 \times 70+0.09 \times 60=80.40 ;
$$

Similarly, $U_{2}=S_{2} \times V=80.80, U_{3}=S_{3} \times V=78.80$ and $U_{4}=S_{4} \times V=78.90$.

The score of the first level evaluation findings is:

$$
U=S \times V=0.37 \times 90+0.33 \times 80+0.22 \times 70+0.08 \times 60=79.90 .
$$

So we can find that the knowledge competitiveness of the PL company was "general", between "general" and "relative strong". The technology and brand factors were "relative strong", the resource and organization factors were "general ",.

\section{Conclusion}

This paper took the evaluation of business knowledge competitiveness as the object of study and built the evaluation model of fuzzy comprehension based on entropy weight. In order to verify the reasonableness of evaluation model, paper took PL company as example of empirical study. The authors got the deeper investigation and research about the knowledge competitiveness of the PL company and designed the reasonable index system, which could make the evaluation results more scientific and objective through adopting the entropy weight evaluation method to enhance the credibility of the weights.

\section{References:}

LI Jin. (2005). The comprehensive evaluation of supply electric enterprise culture construction. North China Electric Power University Journal, 32(5), 108-111.

SU Yong. (2002). The system study about the enterprise culture. Shanghai: Fudan University Press.

ZHOU Jian-guo \& NIU Dong-xiao. (2004). The entropy evaluation of overall strength in the power public company. North China Electric Power University Journal, 31(3), 71-73.

(Edited by Ruby and Chris) 